\title{
Paeonol Inhibits Proliferation of Vascular Smooth Muscle Cells Stimulated by High Glucose via Ras-Raf-ERK1/2 Signaling Pathway in Coculture Model
}

\author{
Junjun Chen, ${ }^{1}$ Min Dai, ${ }^{1,2}$ and Yueqin Wang ${ }^{1}$ \\ ${ }^{1}$ Key Laboratory of Xinian Medicine, Ministry of Education, Anhui Province Key Laboratory of R\&D of Chinese Medicine, \\ Anhui University of Traditional Chinese Medicine, Hefei 230038, China \\ ${ }^{2}$ School of Pharmacy, Anhui University of Traditional Chinese Medicine, Shihe Road 45, Hefei, Anhui 230031, China \\ Correspondence should be addressed to Min Dai; daiminliao@163.com
}

Received 19 February 2014; Revised 29 April 2014; Accepted 18 May 2014; Published 5 June 2014

Academic Editor: Hao Xu

Copyright (C) 2014 Junjun Chen et al. This is an open access article distributed under the Creative Commons Attribution License, which permits unrestricted use, distribution, and reproduction in any medium, provided the original work is properly cited.

\begin{abstract}
Paeonol (Pae) has been previously reported to protect against atherosclerosis (AS) by inhibiting vascular smooth muscle cell (VSMC) proliferation or vascular endothelial cell (VEC) injury. But studies lack how VSMCs and VECs interact when Pae plays a role. The current study was based on a coculture model of VSMCs and VECs to investigate the protective mechanisms of Pae on atherosclerosis (AS) by determining the secretory function of VECs and proliferation of VSMCs focusing on the RasRaf-ERK1/2 signaling pathway. VECs were stimulated by high glucose. Our data showed that high concentration (35.5 mM) of glucose induced damage in VECs. Injury of VECs stimulated VSMC proliferation in the coculture model. Pae $(120 \mu \mathrm{M})$ decreased vascular endothelial growth factor (VEGF) and platelet derivative growth factor B (PDGF-B) release from VECs and inhibited overexpression of Ras, P-Raf, and P-ERK proteins in VSMCs. The results indicate that diabetes modulates the inflammatory response in VECs to stimulate VSMC proliferation and promote the development of AS. Pae was beneficial by inhibiting the inflammatory effects of VECs on VSMC proliferation. This study suggests the inhibitory mechanism of Pae due to the inhibition of VEGF and PDGF-B secretion in VECs and Ras-Raf-ERK1/2 signaling pathway in VSMCs.
\end{abstract}

\section{Introduction}

Atherosclerosis (AS) is a major pathological disease for cardiovascular and cerebrovascular problems and also the most common disease in the cardiovascular system [1]. AS is harmful to human health seriously and responsible for most of the deaths in the senior population [2]. Diabetes mellitus is considered as an important risk factor to the accelerated atherosclerosis [3-5]. High glucose has been shown to injure vascular endothelial cells (VECs) and vascular smooth muscle cells (VSMCs), which are two important cells of artery wall and responsible for AS progression [6]. Among them, VEC inflammatory injury and dysfunction are initial factors, and VSMC proliferation and migration are key pathological features [7-9]. In the native artery, VSMCs are tightly associated with VECs in structure and function. VECs dysfunction will release cytokines like VEGF, bFGF, TGF- $\beta$, and PDGF which have shown regulatory effects on VSMC proliferation and migration.

The mitogen activated protein kinase (MAPK) signaling pathway is one of the most important pathways for cellular stress response to injury. The MAPK signaling pathway consists of four subfamilies: p38 kinases, Jun-NH2terminal kinases (JNK1/2), extracellular signal-regulated kinases (ERK1/2), and ERK5 [10]. Signaling through ERK1/2 is typically initiated by Ras, which can be activated by cytokines like VEGF and PDGF-B [11]. They activate Ras, which directly couples with the Raf (MAPK kinase kinase) and then combines with MEK1/2 (MAPK kinases). MEK1/2 functions as a dual specific kinase, which phosphorylates ERK1/2 directly. High concentrations of glucose (GS) have been shown to induce VEC membrane damage by increasing the release of inflammatory cytokines, such as vascular endothelial growth factor (VEGF) and platelet derived 
<smiles>COc1ccc(C(C)=O)c(O)c1</smiles>

FIgURE 1: Chemical structure of paeonol.

growth factor-B (PDGF-B) which bind with VSMC membrane receptors and lead to the receptor phosphorylation [12-14]. The activated receptors stimulate downstream signal transduction pathways and eventually activate ERK1/2 protein which lead to VSMC proliferation and intimal thickening $[15,16]$. Previous studies have shown that PDGF-B expression was increased when VECs were damaged [17]. PDGF-B then activates the ERK1/2 related signaling pathway, which mainly mediates growth factor-induced cell proliferation $[14,18$, 19]. Statistical analysis also showed that VEGF increased in diabetics and associated with blood glucose concentration which can also activate signal pathway involved in ERK1/2 participate [20]. Accordingly, we hypothesise that high glucose induced VECs producing a series of biologically active substances which stimulated VSMC proliferation.

Paeonol (Pae, 2'-hydroxy-4'-methoxyacetophenone, Figure 1) is the major biologically active compound contained in Cortex Moutan (Paeonia suffruticosa Andrews, Ranunculaceae), which is a Chinese herbal remedy widely used in clinical treatment of inflammatory diseases such as atopic dermatitis, hyperlipidemia, and atherosclerosis $[21,22]$. Our previous investigations suggested that Pae had a significant effect on different aspects of AS. In vivo, Pae prevented AS in our experimental model and protected against arterial endothelial cell hyperlipidemia [21, 23, 24]. In vitro, serum containing Pae significantly inhibited TNF$\alpha$-induced VSMC proliferation. Furthermore, our most current research confirmed that Pae significantly reduced the phosphorylation levels of JNK1/2, p38, and ERK1/2, which was activated by TNF- $\alpha$, ox-LDL, and/or bacterial lipopolysaccharide in VECs [25]. Unfortunately, the previous in vitro studies were based only on a single cell type (VECs or VSMCs) and ignored the interactions between these two important cell types.

The current study was focused on a coculture model to determine essential crosstalk pathways between VECs and VSMCs. The coculture model is a novel method which could simulate the environment in native artery to study Pae action. The principal aim of this study was to investigate the effects of Pae on VECs cellular damage and its downstream effects on VSMC proliferation, which ultimately leads to the pathological feature observed in AS. In addition, this study worked to determine the relationship between the secretion function of damaged VECs and the Ras-RafERK1/2 signaling pathway in VSMCs in order to clarify the therapeutic mechanisms of Pae. This experiment model provided a theoretical basis for Pae intervention in AS and optimized a technical platform to determine the cellular target of novel therapeutic compounds.

\section{Materials and Methods}

2.1. Chemicals and Reagents. The compound paeonol (99\% purity) was obtained from Baicao Plants Biotech Co., Ltd. (Anhui, China). Dulbecco's modified Eagle's medium (DMEM), Transwell chamber, type I collagenase, and fetal bovine serum (FBS) were purchased from Gibco Life Technologies, Co., Ltd. (Paisley, UK). 3-(4,5-dimethylthiazolyl2)-2,5-diphenyltetrazolium bromide (MTT) was obtained from Sigma Chemical Co. (St. Louis, MO, USA). Lactic dehydrogenase $(\mathrm{LDH})$ reagent was purchased from Nanjing Jiancheng Bioengineering Institute (Nanjing, China). Antibodies against PDGF-B, Ras, Raf, phosphorylated Raf (P-Raf), ERK1/2, and phosphorylated ERK1/2 (P-ERK1/2) were obtained from Cell Signaling Technology (Beverly, MA, USA). PDGFR inhibitor (Sunitinib Malate) and ERK1/2 inhibitor (PD98059) were purchased from Santa Cruz Biotechnology Co. (Santa Cruz, CA, USA).

2.2. Animals. Sprague-Dawley (SD) rats $(160 \pm 10 \mathrm{~g})$ were obtained from Shanghai Super-B\&K Laboratory Animal Corp. Ltd. (license number: SCXK 2008-0016). All animal protocols were conducted in accordance with animal welfare protocols at the local institution Animal Care and Use Committee.

2.3. Cell Culture. VECs and VSMCs were isolated from rat thoracic aortas by primary explants techniques according to a previously published protocol $[26,27]$. Briefly, the cells were incubated in a $50 \mathrm{~mL}$ culture flask at $37^{\circ} \mathrm{C}$ in a humidified atmosphere containing $5 \% \mathrm{CO}_{2}$. Culture medium was composed of DMEM supplemented with $20 \% \mathrm{FBS}, \mathrm{NaHCO}_{3}$ $(1.8 \mathrm{~g} / \mathrm{L})$, penicillin $100 \mathrm{kU} / \mathrm{L}$, and gentamicin $100 \mathrm{kU} / \mathrm{L}$. The culture media were changed every $3 \mathrm{~d}$. Cells were grown to an $80 \%$ confluence state and subcultured using $0.2 \%$ trypsin. VECs and VSMCs at passages three to five were used in the current study.

2.4. VECs and VSMCs Coculture. The coculture model was created to investigate the effects of damaged VECs on VSMCs through polycarbonate filter membrane (Transwell chamber). The coculture model was created according to the methods of Fillinger et al. [28, 29]. VECs and VSMCs were diluted into cell suspension of $1 \times 10^{5}$ cells $/ \mathrm{mL}$. VECs were inoculated into the bottom of a 6-well chamber and pretreated with a high glucose $(\mathrm{HG})$ concentration $(35.5 \mathrm{mM})$ for $48 \mathrm{~h}$, whereas $5.5 \mathrm{mM}$ was considered as normal glucose concentration. Then, VSMCs were inoculated into the top of the Transwell plate. The Transwell chamber was then set into the 6-well chamber and cocultured. The two types of cells were not physically connected but were able to interact by secreting soluble factors through a polycarbonate filter membrane. 
2.5. Cell Survival Rate Assay. The cytotoxic effects of glucose and Pae on VECs growth were determined through the 3(4,5-dimethylthiazolyl-2)-2,5-diphenyltetrazolium bromide (MTT) assay. VECs were grown to $80 \%$ confluence and then seeded into a 96-well flat-bottom plate and incubated with DMEM supplemented with 20\% FBS. Different concentrations of glucose $(5.5,15.5,25.5,35.5$, and $45.5 \mathrm{mM})$ at multiple time points $(0,12,24,48$, and $72 \mathrm{~h})$ were used. Furthermore, to investigate the effects of Pae on VECs, cells were pretreated with different concentrations of Pae $(7.5,15$, $30,60$, and $120 \mu \mathrm{M})$ for different time points $(0.5,6,12$, and $24 \mathrm{~h}$ ) before being stimulated by glucose at a suitable concentration $(35.5 \mathrm{mM})$ for $48 \mathrm{~h}$. Cells were incubated with glucose at $5.5 \mathrm{mM}$, which is indicated as the normal glucose (NG) group. After treatment, cells were incubated with MTT (20 $\mu \mathrm{L} /$ well) (Sigma Chemical, USA) for $4 \mathrm{~h}$ at $37^{\circ} \mathrm{C}$. The medium was then removed and DMSO $(200 \mu \mathrm{L} /$ well $)$ was added to solubilize the precipitate. The absorbance then was measured at $490 \mathrm{~nm}$ on an absorbance microplate reader (Molecular Devices, USA).

2.6. Lactic Dehydrogenase (LDH) Release Assay. The LDH release was used to investigate the cytoprotective effects of Pae on VEC injury due to high glucose concentrations. VECs were centrifuged at $160 \times \mathrm{g}$ for $8 \mathrm{~min}$ to obtain a supernatant comprised of extracellular VEC components. VECs were incubated with $2 \%$ Triton X-100, freezed and thawed three times, and centrifuged at $240 \times \mathrm{g}$ for $5 \mathrm{~min}$ to obtain a supernatant comprised of intracellular components. $\mathrm{LDH}$ concentrations in the extracellular medium and intracellular medium were quantified through the LDH reagent for clinical diagnosis.

2.7. Immunocytochemistry Assay. VECs seeded on coverslips were rinsed with ultrapure water twice, fixed with acetone for $20 \mathrm{~min}$, and then rinsed with phosphate-buffered saline (PBS). Cells were soaked in $3 \% \mathrm{H}_{2} \mathrm{O}_{2}$ at room temperature for 30 min. After washing with PBS, blocking buffer containing $1 \%$ goat serum was added and incubated for $20 \mathrm{~min}$. The primary antibody (1:200 diluted) against VEGF or PDGF$\mathrm{B}$ was added and incubated at $4^{\circ} \mathrm{C}$ overnight. A biotinylated secondary antibody was incubated for $20 \mathrm{~min}$; then Streptavidin/Peroxidase (SP) reagent was incubated for $20 \mathrm{~min}$ at room temperature. The slides were colored with DAB under a light microscope for $10 \mathrm{~min}$ before examination. Immunocomplexes were visualized by the $\mathrm{DAB}$ detection system. Brown or dark brown stained cells were considered positive cells. Ten randomly selected fields were visualized at 200x magnification.

2.8. Western Blotting Analysis. Cytoplasmic proteins were obtained using a cell lysis buffer (20 mM HEPEs, $2 \mathrm{mM}$ $\mathrm{MgCl}_{2}, 1 \mathrm{mM}$ EDTA, $2 \mathrm{mM}$ DTT, $1 \mathrm{mM}$ PMSF, pH 7.4) and stored at $-80^{\circ} \mathrm{C}$. The protein concentrations were quantified by the BCA method. Aliquots $(30 \mu \mathrm{L})$ were separated on a $10 \%$ SDS-PAGE and transferred to an equilibrated polyvinylidene difluoride membrane (PVDF) by electroblotting. Membranes were blocked in 5\% fat-free milk for $2 \mathrm{~h}$ at room

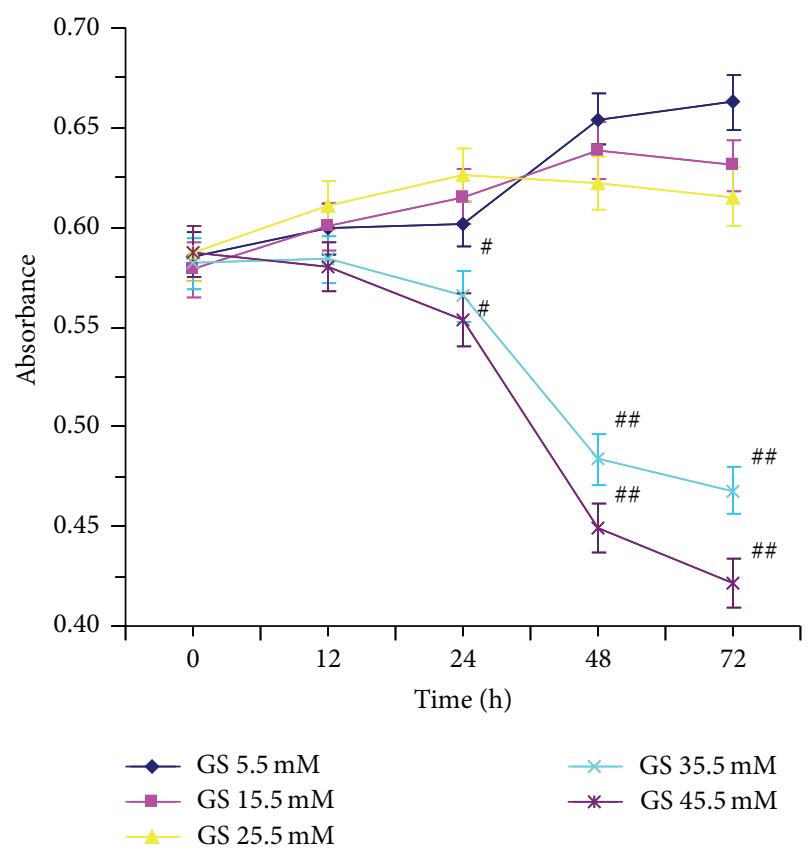

FIGURE 2: Effect of high glucose on VECs injury. VECs were induced by various concentrations $(5.5,15.5,25.5,35.5$, and $45.5 \mathrm{mM})$ of glucose and incubated for different time points $(0,12,24,48$, and $72 \mathrm{~h}$ ) to explore the effect of glucose on VECs.

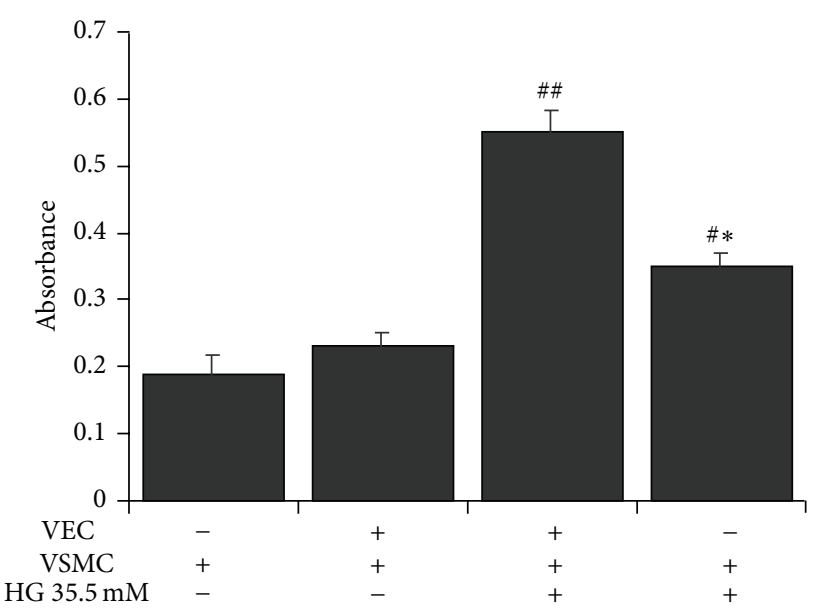

FIGURE 3: Effect of high glucose on VSMC proliferation in the coculture model. VECs were pretreated with high glucose concentration $(35.5 \mathrm{mM})$ for $48 \mathrm{~h}$ and then cocultured with VSMCs for another $24 \mathrm{~h}$ to stimulate VSMC proliferation. ${ }^{\#} P<0.05$, ${ }^{\#} P<0.01$ versus NG $(5.5 \mathrm{mM})$ coculture group; ${ }^{*} P<0.05$ versus HG $(35.5 \mathrm{mM})$ coculture group.

temperature and incubated at $4^{\circ} \mathrm{C}$ overnight with primary antibodies (rabbit anti-Ras, rabbit anti-Raf, rabbit antiphospho-Raf, mouse anti-ERK1/2, and rabbit anti-phosphoERK1/2). Horseradish peroxidase labeled secondary antibodies were added and incubated at room temperature for $2 \mathrm{~h}$. Bands were detected by enhanced chemiluminescence (ECL) kit. Beta-actin protein levels were used as an endogenous control to allow the normalization of target proteins. 


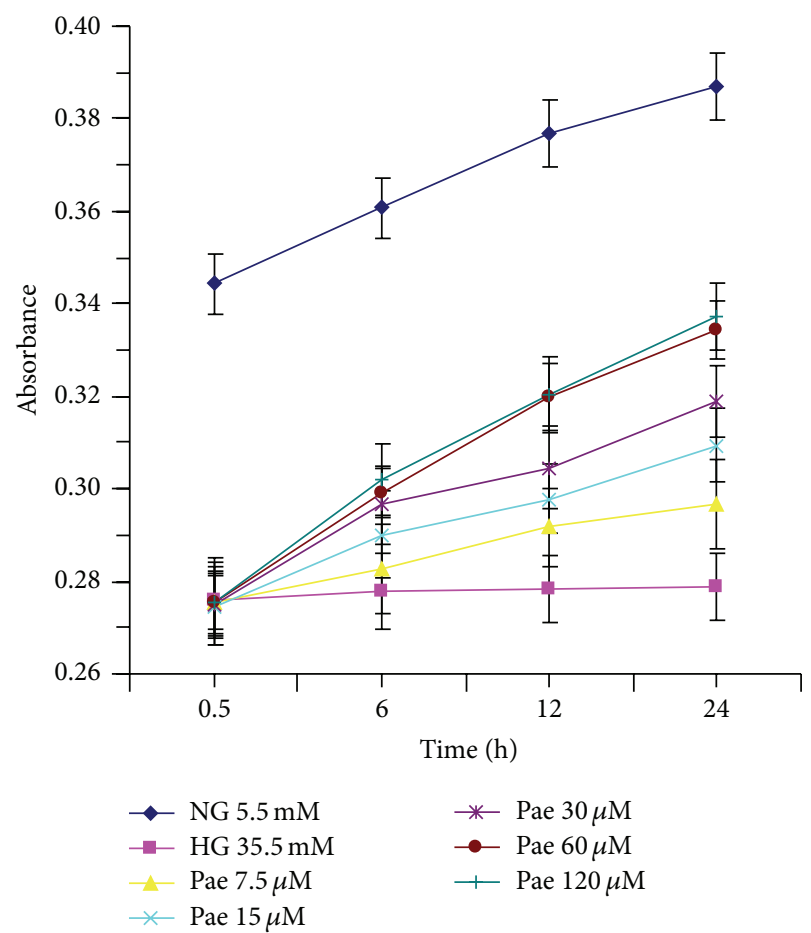

(a)

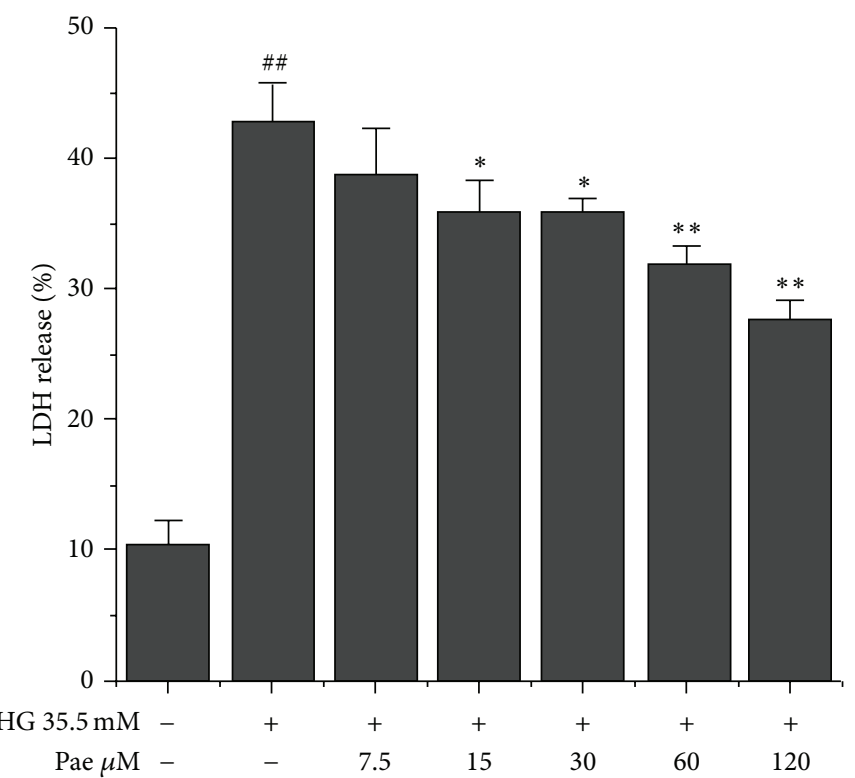

(b)

FIGURE 4: Effect of paeonol on survival and LDH release in VECs. VECs survival (a) and LDH release (b) induced by high glucose (35.5 mM) were determined by MTT assay. ${ }^{\# \#} P<0.01$ versus NG $(5.5 \mathrm{mM})$ group; ${ }^{*} P<0.05,{ }^{* *} P<0.01$ versus HG (35.5 mM) group.

The band intensity was quantified and analyzed with Quantity One software (Bio-Rad, USA). The results were quantified by using the integrated optical density of each band with the background subtracted.

2.9. Statistical Analysis. Data were analyzed by a one-way ANOVA or independent $t$-test. Data are presented as mean \pm S.D. averaging three or more independent experiments. Significance was noted at a $P<0.05$.

\section{Results}

3.1. High Glucose Injured VECs. VEC survival was measured by MTT assay. VEC survival rates in glucose concentrations of 5.5 and $15.5 \mathrm{mM}$ increased in a dose- and time-dependent manner. When the concentration was up to $25.5 \mathrm{mM}$, VEC survival rate was a little inhibited after incubatation for $48 \mathrm{~h}$. However, the rate was inhibited significantly in glucose concentrations of 35.5 and $45.5 \mathrm{mM}$ even in $24 \mathrm{~h}$ incubation. VEC morphology changed into contraction, rounded and smaller in glucose concentrations of 35.5 and $45.5 \mathrm{mM}$. Moreover, cells cultured in glucose concentrations of $35.5 \mathrm{mM}$ for $48 \mathrm{~h}$ showed the most significant inhibition (Figure 2).

3.2. High Glucose Induced VSMC Proliferation in the Coculture Model. VECs were pretreated with a high glucose (HG) concentration $(35.5 \mathrm{mM})$ for $48 \mathrm{~h}$ and then cocultured with
VSMCs for $24 \mathrm{~h}$ to induce VSMC proliferation. Glucose concentration of $5.5 \mathrm{mM}$ was considered as normal group. VSMCs inoculated in the upper chamber alone and stimulated with $35.5 \mathrm{mM}$ glucose were set as the single cultured group. Compared with the normal group, VSMCs proliferated significantly in the cocultured group $(P<0.01)$, as well as in the single cultured group $(P<0.05)$. Compared with the single cultured group, VSMCs in the coculture group also proliferated significantly $(P<0.05)$, which suggested that injured VECs stimulated VSMCs proliferation (Figure 3). These results showed that VSMC proliferation stimulated by high glucose injured VECs was even much stronger than that stimulated directly by high glucose.

3.3. Paeonol Partially Restored Survival and Reduced LDH Release in VECs. VECs were pretreated with Pae at 7.5, 15, 30,60 , and $120 \mu \mathrm{M}$ for $24 \mathrm{~h}$ prior to being stimulated by high glucose. Compared with control group, VEC survival increased in a dose- and time-dependent manner. The effect of Pae was greatest $(P<0.01)$ when VECs were pretreated with Pae at $120 \mu \mathrm{M}$ (Figure 4(a)). Additionally, the LDH level was increased by the stimulation of $35.5 \mathrm{mM}$ glucose. $\mathrm{LDH}$ release was gradually reduced as the Pae concentration increased. At a final concentration of $15 \mu \mathrm{M}$ Pae, $\mathrm{LDH}$ release was significantly decreased $(P<0.05)$. When the concentration of Pae was $60 \mu \mathrm{M}$ and $120 \mu \mathrm{M}$, the difference in $\mathrm{LDH}$ release was highly significant $(P<0.01)$ (Figure $4(\mathrm{~b}))$. 


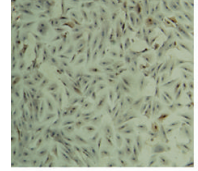

(A)

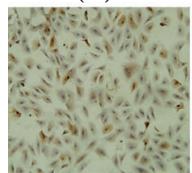

(D)

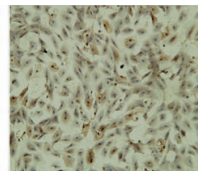

(B)

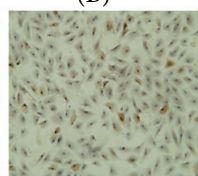

(E)

(a)

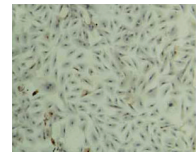

(A)

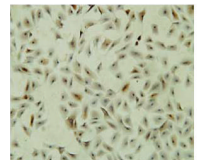

(D)

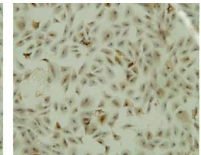

(B)

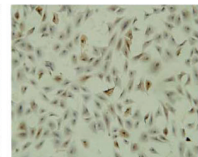

(E)

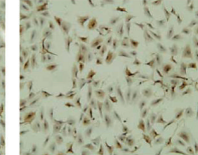

(C)

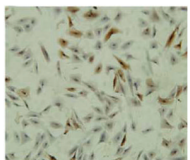

(F) (c)

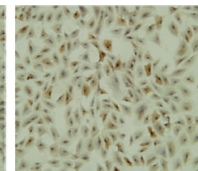

(C)

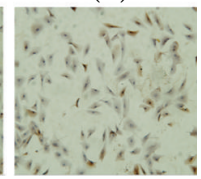

(F)

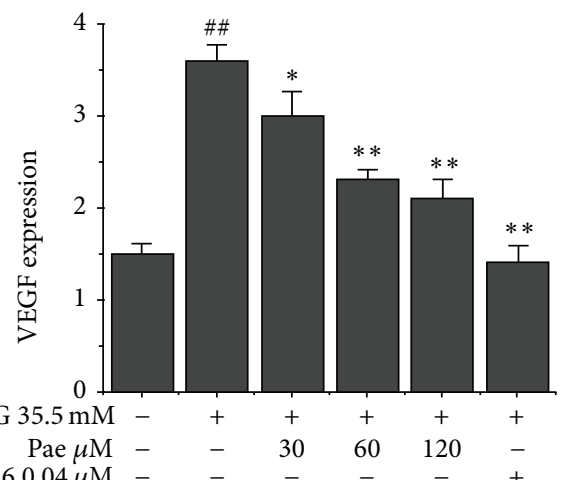

(b)

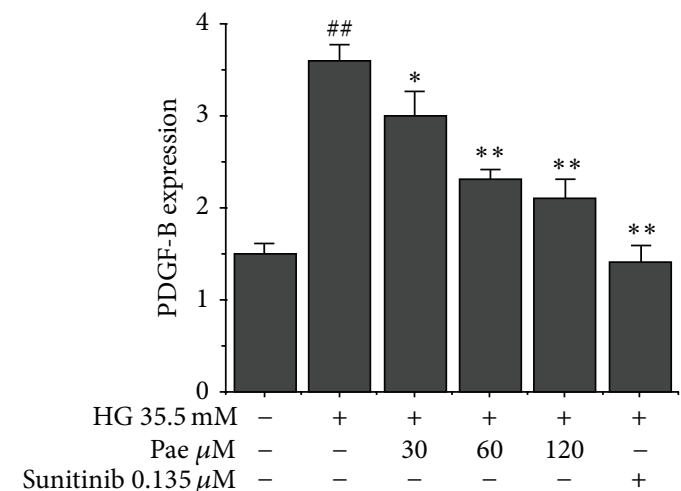

(d)

FIGURE 5: Effect of paeonol on VEGF and PDGF-B expression in VECs. VEGF (a and b) and PDGF-B (c and d) expression in VECs was performed through immunocytochemical staining as described in Section 2. (A) NG (5.5 mM); (B) HG (35.5 mM); (C) HG + Pae (30 $\mu \mathrm{M})$; (D) HG + Pae $(60 \mu \mathrm{M})$; (E) HG + Pae $(120 \mu \mathrm{M})$; (F) HG + Sunitinib $(0.135 \mu \mathrm{M}) .{ }^{\# \#} P<0.01$ versus NG $(5.5 \mathrm{mM})$ group; ${ }^{*} P<0.05,{ }^{* *} P<0.01$ versus HG (35.5 mM) group.

3.4. Paeonol Inhibited VEGF and PDGF-B Overexpression in VECs. Through immunocytochemical staining, VEGF and PDGF-B expression were quantified inside VECs. Positive cytoplasm was brown. The positive rate of VEC was used to evaluate the effect of high glucose and Pae. High glucose resulted in increasing expression of VEGF and PDGF-B in vascular endothelial cells. The expression decreased with increasing concentrations of Pae (Figures 5(a) and 5(c)). Concentrations of $60 \mu \mathrm{M}$ and $120 \mu \mathrm{M}$ Pae decreased the expression of VEGF and PDGF-B significantly $(P<0.01)$. As shown in Figures 5(b) and 5(d), the VEGFR inhibitor, SU5416, not only decreased the expression of VEGF significantly $(P<0.01)$ but also decreased the number of cells with brown nuclei. The PDGFR inhibitor, Sunitinib, showed the same results. These results suggested that the proliferation of VSMC induced by high glucose may be achieved by upregulating VEGF and PDGF-B factors. And Pae reversed the effect of high glucose. Based on the results, we chose an appropriate signaling pathway to further explore how the cytokine mediates proliferation of VSMC.

3.5. Paeonol Blocked Ras-Raf-ERK1/2 Pathway in VSMCs in Coculture Model. One of the most important downstream signaling cascades of VEGF and PDGF-B is the Ras-RafERK pathway [30]. To inhibit this signaling pathway initially, SU5416 and Sunitinib were used to inhibit the respective receptor and associated downstream proteins. In order to explore the cellular target of Pae, PD98059 was used to inhibit ERK1/2 protein expression. As shown in Figure 6, high glucose $(35.5 \mathrm{mM})$ significantly induced the expression of Ras, P-Raf, and P-ERK1/2 in VSMCs $(P<0.01)$. Pae $(120 \mu \mathrm{M})$ significantly inhibited the expression of Ras, PRaf, and P-ERK1/2 $(P<0.05)$. For protein Ras and P-Raf, SU5416 and Sunitinib significantly reduced the expression $(P<0.01)$. Likewise, cells treated with SU5416, Sunitinib, or PD98059 showed significantly inhibitory effect on P-ERK1/2 expression. Moreover, the cotreatment of Pae $(120 \mu \mathrm{M})$ with SU5416, Sunitinib, or PD98059 had a greater inhibitory effect than treating VSMCs with each individual compound. These results indicated that the effect of Pae to block Ras-Raf-ERK signaling pathway may be due to the ability to inhibit the expression of VEGF and PDGF-B.

3.6. Paeonol Inhibited VSMC Proliferation in Coculture Model. VECs were pretreated with different concentrations of 

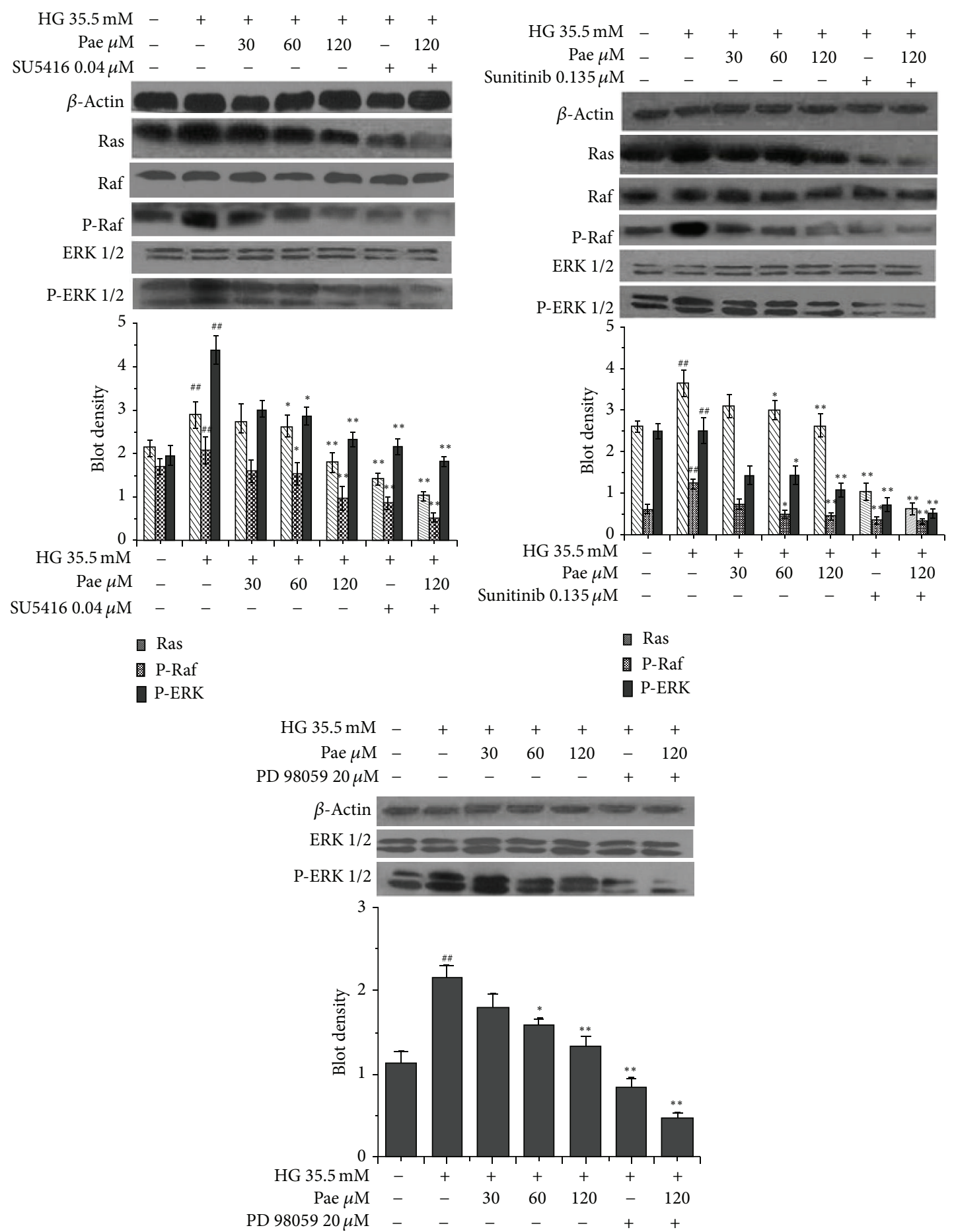

FIGURE 6: Effect of paeonol on Ras-Raf-ERK1/2 signaling pathway in VSMCs in the coculture model. VECs were pretreated with various concentrations of Pae for $24 \mathrm{~h}$ before being incubated with HG (final concentration of $35.5 \mathrm{mM}$ ) for another $48 \mathrm{~h}$. Then VSMCs were inoculated into the top of the Transwell plate and cocultured with VECs for another $24 \mathrm{~h}$. Protein concentrations were analyzed by Western blot. ${ }^{\# \#} P<0.01$ versus NG $(5.5 \mathrm{mM})$ coculture group; ${ }^{*} P<0.05,{ }^{* *} P<0.01$ versus $\mathrm{HG}(35.5 \mathrm{mM})$ coculture group.

Pae $(30,60$, and $120 \mu \mathrm{M})$ before being stimulated by high glucose $(35.5 \mathrm{mM})$ for $48 \mathrm{~h}$. The results of MTT indicated that Pae $(30,60$, and $120 \mu \mathrm{M})$ inhibited VSMC proliferation $(P<$ $0.01)$ compared with high glucose group. The levels of VSMC proliferation were also inhibited with Pae plus Sunitinib or
PD98059 group. The level of inhibition was greater than each single treatment group, but no significant difference was observed (Figure 7). The results proved the protective effect of Pae on VSMC proliferation which cocultured with VECs in high glucose. 


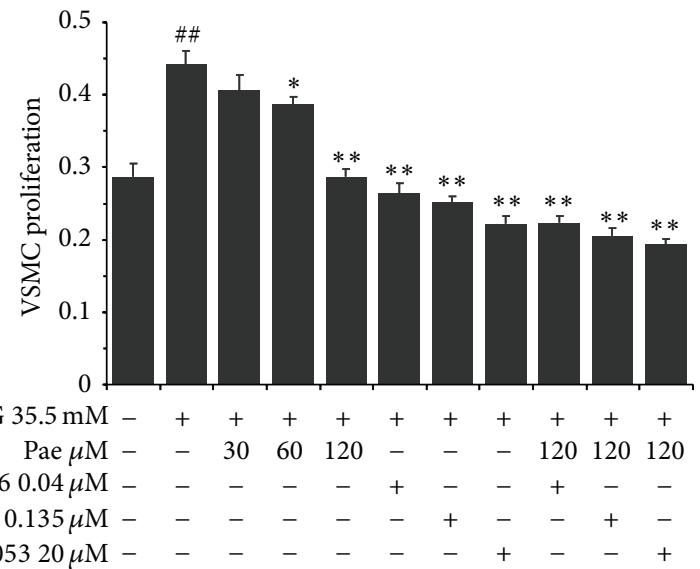

FIGURE 7: Effect of paeonol on VSMC proliferation in the coculture model. VECs and VSMCs cocultured model was established as described in Section 2. The effects of paeonol on VSMC proliferation were assayed by MTT. ${ }^{\#} P<0.01$ versus NG $(5.5 \mathrm{mM})$ coculture group; ${ }^{*} P<0.05,{ }^{* *} P<0.01$ versus $\mathrm{HG}(35.5 \mathrm{mM})$ coculture group.

\section{Discussion}

Diabetes is one of the main predisposing factors of atherosclerosis. Type 2 diabetic vascular complications are based on diabetes-induced arteriosclerosis [31]. High glucose concentrations exerted a harmful effect on VECs and VSMCs, which are important components of artery wall. Impaired VECs destroy the structure of the endothelial monolayer and lead to vascular barrier dysfunction [32]. Large numbers of damaged VECs have been shown to impact the vasodilatation function and VECs regeneration [33]. These effects may be an important mechanism of diabetic complication of AS [34]. Our present study indicated that high glucose concentrations increased membrane permeability and decreased VEC survival. VSMC proliferation and phenotypic transformation are critical pathological features of AS, which depend on growth factors, cytokines, and vasoactive substances. VEGF is the initial factor for early VEC angiogenesis and VSMC proliferation. However, a mature vascular system can not be generated alone. PDGF-B also had a strong effect on promoting inflammation in this study. Several reports support the hypothesis that PDGF-B plays a crucial role in the development of atherosclerotic plaques [35-37]. Our study demonstrated that VEGF and PDGF-B expression in VECs was increased significantly by high glucose. Treatment with Pae significantly attenuated VEGF and PDGF-B expression. Therefore, our results indicate that Pae may act against VEC injury by reducing VEGF and PDGF-B release as an antiinflammatory and antithromboembolic effect.

Monolayer cell culture, in many cases, is difficult to simulate the interaction between various types of cells in vivo. Coculture model can simulate the internal in vivo environment to observe important interactions between cells [38]. In addition to the morphological changes, a variety of angiogenesis-related gene expression changes occur in VECs cocultured with VSMCs $[39,40]$. Thus, the coculture model in this study not only retained the cellular microenvironment of material and structural basis in vivo but also showed the advantages of controllability and macroscopic visibility of the cell culture. The significant proliferation of VSMCs in the coculture model confirmed that damaged VECs could stimulate VSMC proliferation and also indicated that basic interaction between VECs and VSMCs can be carried out smoothly. Accordingly, the coculture model provides the necessary conditions to explore the pathogenesis of atherosclerosis.

Ras-Raf-ERK1/2 signaling pathway was activated by high glucose concentration in our coculture model. The phosphorylated proteins regulate the target gene expression which promote cell proliferation [41, 42]. Our study indicated that Pae protected the integrity and survival rate of VECs and reduced the VEGF and PDGF-B release of VECs into the cocultured model to inhibit VSMC proliferation. We also presume that the effect may relate to the downstream signaling pathway in VSMCs. For this reason, the study used the VEGFR and PDGFR inhibitor to explore the possible targets of Pae. The results showed that Pae in combination with SU5416 or Sunitinib could further reduce the Ras, PRaf, and P-ERK1/2 expression levels. In order to further clarify whether Pae had effect on protein ERK1/2 as well, the ERK1/2 inhibitor, PD98059, was utilized. We found that ERK1/2 expression levels were reduced when treated with Pae and the inhibitor. These findings indicated that Pae may decrease the VEGF and PDGF-B expression, resulting in protecting the VEC and inhibiting the downstream signaling pathway. Otherwise, Pae may inhibit the VSMC proliferation due to the direct inhibition of Ras-Raf-ERK1/2 pathway.

Our findings confirmed that Pae can inhibit the proliferation of VSMC cocultured with VECs induced by high glucose concentration. One reason is that Pae decreased the release of inflammatory cytokines, VEGF and PDGF-B, in VECs which combined with VSMC membrane receptors and led to phosphorylation of the receptors. The inhibited receptors blocked downstream signaling pathways, Ras-RafERK1/2, which are responsible for VSMC proliferation. The other is that Pae inhibited Ras-Raf-ERK1/2 activation directly to suppress VSMC proliferation. Based on our study, Pae appears to be a promising inhibitor to AS.

\section{Conclusion}

Taken together, our findings indicated that the mechanism of Pae effects on AS might involve its sequential inhibition of VEGF and PDGF-B in VECs and the Ras-Raf-ERK1/2 signaling pathways in VSMCs.

\section{Conflict of Interests}

The authors claim no conflict of interests involved in the study.

\section{Acknowledgments}

This work was supported by research Grants from the National Natural Science Foundation of China (81073090 and 
81274134). The authors thank Dr. David J. Blake for critical reading of this paper.

\section{References}

[1] A. Didangelos, D. Simper, C. Monaco, and M. Mayr, "Proteomics of acute coronary syndromes," Current Atherosclerosis Reports, vol. 11, no. 3, pp. 188-195, 2009.

[2] J. C. Wang and M. Bennett, "Aging and atherosclerosis: mechanisms, functional consequences, and potential therapeutics for cellular senescence," Circulation Research, vol. 111, no. 2, pp. 245-259, 2012.

[3] J. A. Beckman, M. A. Creager, and P. Libby, "Diabetes and atherosclerosis epidemiology, pathophysiology, and management," Journal of the American Medical Association, vol. 287, no. 19, pp. 2570-2581, 2002.

[4] L. Piconi, L. Quagliaro, R. Assaloni et al., "Constant and intermittent high glucose enhances endothelial cell apoptosis through mitochondrial superoxide overproduction," Diabetes/Metabolism Research and Reviews, vol. 22, no. 3, pp. 198203, 2006.

[5] S. M. Baumgartner-Parzer, L. Wagner, M. Pettermann, J. Grillari, A. Gessl, and W. Waldhausl, "High-glucose-triggered apoptosis in cultured endothelial cells," Diabetes, vol. 44, no. 11, pp. 1323-1327, 1995.

[6] S. Stintzing, M. Ocker, A. Hartner, K. Amann, L. Barbera, and D. Neureiter, "Differentiation patterning of vascular smooth muscle cells (VSMC) in atherosclerosis," Virchows Archiv, vol. 455, no. 2, pp. 171-185, 2009.

[7] A. R. Keramati, R. Singh, A. Lin et al., "Wild-type LRP6 inhibits, whereas atherosclerosis-linked LRP6R611C increases PDGF-dependent vascular smooth muscle cell proliferation," Proceedings of the National Academy of Sciences of the United States of America, vol. 108, no. 5, pp. 1914-1918, 2011.

[8] K. Sternberg, M. Gratz, K. Koeck et al., "Magnesium used in bioabsorbable stents controls smooth muscle cell proliferation and stimulates endothelial cells in vitro," Journal of Biomedical Materials Research B, Applied Biomaterials, vol. 100, no. 1, pp. 41-50, 2012.

[9] C. Qin and Z. Liu, "In atherogenesis, the apoptosis of endothelial cell itself could directly induce over-proliferation of smooth muscle cells," Medical Hypotheses, vol. 68, no. 2, pp. 275-277, 2007.

[10] T. P. Garrington and G. L. Johnson, "Organization and regulation of mitogen-activated protein kinase signaling pathways," Current Opinion in Cell Biology, vol. 11, no. 2, pp. 211-218, 1999.

[11] J. Huang, L.-S. Li, D.-L. Yang, Q.-H. Gong, J. Deng, and X.N. Huang, "Inhibitory effect of ginsenoside Rg1 on vascular smooth muscle cell proliferation induced by PDGF-BB is involved in nitric oxide formation," Evidence-Based Complementary and Alternative Medicine, vol. 2012, Article ID 314395, 12 pages, 2012.

[12] L. Labrecque, S. Lamy, A. Chapus et al., "Combined inhibition of PDGF and VEGF receptors by ellagic acid, a dietary-derived phenolic compound," Carcinogenesis, vol. 26, no. 4, pp. 821-826, 2005.

[13] H. Shimizu, Y. Nakagawa, C. Murakami, N. Aoki, S. KimMitsuyama, and H. Miyazaki, "Protein tyrosine phosphatase PTPEM negatively regulates PDGF $\beta$-receptor signaling induced by high glucose and PDGF in vascular smooth muscle cells," The American Journal of Physiology-Cell Physiology, vol. 299, no. 5, pp. C1144-C1152, 2010.
[14] M. Kernt, A. S. Neubauer, R. G. Liegl et al., "Sorafenib prevents human retinal pigment epithelium cells from light-induced overexpression of VEGF, PDGF and PlGF," The British Journal of Ophthalmology, vol. 94, no. 11, pp. 1533-1539, 2010.

[15] J. Ortmann, M. Veit, S. Zingg et al., "Estrogen receptor- $\alpha$ but not $-\beta$ or GPER inhibits high glucose-induced human VSMC proliferation: potential role of ROS and ERK," Journal of Clinical Endocrinology and Metabolism, vol. 96, no. 1, pp. 220-228, 2011.

[16] M. G. Sirois, M. Simons, and E. R. Edelman, "Antisense oligonucleotide inhibition of PDGFR- $\beta$ receptor subunit expression directs suppression of intimal thickening," Circulation, vol. 95, no. 3, pp. 669-676, 1997.

[17] W. Lederle, N. Linde, J. Heusel et al., "Platelet-derived growth factor-B normalizes micromorphology and vessel function in vascular endothelial growth factor-A-induced squamous cell carcinomas," The American Journal of Pathology, vol. 176, no. 2, pp. 981-994, 2010.

[18] S. M. Wilhelm, L. Adnane, P. Newell, A. Villanueva, J. M. Llovet, and M. Lynch, "Preclinical overview of sorafenib, a multikinase inhibitor that targets both Raf and VEGF and PDGF receptor tyrosine kinase signaling," Molecular Cancer Therapeutics, vol. 7, no. 10, pp. 3129-3140, 2008.

[19] A. Das, U. Shergill, L. Thakur et al., "Ephrin B2/EphB4 pathway in hepatic stellate cells stimulates Erk-dependent VEGF production and sinusoidal endothelial cell recruitment," The American Journal of Physiology-Gastrointestinal and Liver Physiology, vol. 298, no. 6, pp. G908-G915, 2010.

[20] S. Satofuka, A. Ichihara, N. Nagai et al., "(Pro)renin receptormediated signal transduction and tissue renin-angiotensin system contribute to diabetes-induced retinal inflammation," Diabetes, vol. 58, no. 7, pp. 1625-1633, 2009.

[21] H. Li, M. Dai, and W. Jia, "Paeonol attenuates high-fat-dietinduced atherosclerosis in rabbits by anti-inflammatory activity," Planta Medica, vol. 75, no. 1, pp. 7-11, 2009.

[22] J. Hu, Y.-L. Li, Z.-L. Li et al., "Chronic supplementation of paeonol combined with danshensu for the improvement of vascular reactivity in the cerebral basilar artery of diabetic rats," International Journal of Molecular Sciences, vol. 13, no. 11, pp. 14565-14578, 2012.

[23] M. Dai, Q. Y. Liu, C. G. Gu, and H. Y. Zhang, "Inhibitory effect of paeonol on lipid peroxidational reaction and oxidational decorate of low density lipoprotein," China Journal of Chinese Materia Medica, vol. 25, no. 10, pp. 625-627, 2000.

[24] M. Dai, X. Zhi, D. Peng, and Q. Liu, "Inhibitory effect of paeonol on experimental atherosclerosis in quails," China Journal of Chinese Materia Medica, vol. 24, no. 8, pp. 488-512, 1999.

[25] Y.-Q. Wang, M. Dai, J.-C. Zhong, and D.-K. Yin, "Paeonol inhibits oxidized low density lipoprotein-induced monocyte adhesion to vascular endothelial cells by inhibiting the mitogen activated protein kinase pathway," Biological and Pharmaceutical Bulletin, vol. 35, no. 5, pp. 767-772, 2012.

[26] L.-L. Pan, M. Dai, and W. Wang, "A new method for culturing endothelial cells from the rat aorta," Chinese Pharmacological Bulletin, vol. 23, no. 3, pp. 410-413, 2007.

[27] W. Wang, M. Dai, and L.-L. Pan, "A new method for culturing vascular smooth muscle cells from the rabbit aorta," Chinese Pharmacological Bulletin, vol. 24, no. 11, pp. 1534-1537, 2008.

[28] M. F. Fillinger, L. N. Sampson, J. L. Cronenwett, R. J. Powell, and R. J. Wagner, "Coculture of endothelial cells and smooth muscle cells in bilayer and conditioned media models," Journal of Surgical Research, vol. 67, no. 2, pp. 169-178, 1997. 
[29] N. S. Roy, C. Cleren, S. K. Singh, L. Yang, M. F. Beal, and S. A. Goldman, "Functional engraftment of human ES cell-derived dopaminergic neurons enriched by coculture with telomeraseimmortalized midbrain astrocytes," Nature Medicine, vol. 12, no. 11, pp. 1259-1268, 2006.

[30] H. Zhang, X. Jia, F. Han et al., "Dual-delivery of VEGF and PDGF by double-layered electrospun membranes for blood vessel regeneration," Biomaterials, vol. 34, no. 9, pp. 2202-2212, 2013.

[31] J. A. Beckman, F. Paneni, F. Cosentino, and J. A. Beckman, "Diabetes and vascular disease: pathophysiology, clinical consequences, and medical therapy: part II," European Heart Journal, vol. 108, no. 13, pp. 1527-1532, 2013.

[32] E. Stoyanova, M. Trudel, H. Felfly, W. Lemsaddek, D. Garcia, and G. Cloutier, "Vascular endothelial dysfunction in $\beta$ Thalassemia occurs despite increased eNOS expression and preserved vascular smooth muscle cell reactivity to NO," PLoS ONE, vol. 7, no. 6, Article ID e38089, 2012.

[33] J. S. Petrofsky, "The effect of type-2-diabetes-related vascular endothelial dysfunction on skin physiology and activities of daily living," Journal of Diabetes Science Technology, vol. 5, no. 3, pp. 657-667, 2011.

[34] S. Sartini, S. Cosconati, L. Marinelli et al., "Benzofuroxane derivatives as multi-effective agents for the treatment of cardiovascular diabetic complications. Synthesis, functional evaluation, and molecular modeling studies," Journal of Medicinal Chemistry, vol. 55, no. 23, pp. 10523-10531, 2012.

[35] J. Homsi and A. I. Daud, "Spectrum of activity and mechanism of action of VEGF/PDGF inhibitors," Cancer Control, vol. 14, no. 3, pp. 285-294, 2007.

[36] C. Ionescu, I. Berindan-Neagoe, C. Burz et al., "The clinical implications of platelet derived growth factor B, vascular endothelial growth factor and basic fibroblast growth factor in colorectal cancer," Journal of Buon, vol. 16, no. 2, pp. 274-276, 2011.

[37] N. Liu, J.-T. Liu, Y.-Y. Ji, and P.-P. Lu, "Effects and mechanisms of the functional parts of Dahuang Zhechong Pill containing serum on platelet-derived growth factor-stimulated proliferation of vascular smooth muscle cells," Chinese Journal of Integrative Medicine, vol. 19, no. 6, pp. 432-438, 2013.

[38] D. Ge, N. Meng, L. Su et al., "Human vascular endothelial cells reduce sphingosylphosphorylcholine-induced smooth muscle cell contraction in co-culture system through integrin B4 and Fyn," Acta Pharmacologica Sinica, vol. 33, no. 1, pp. 57-65, 2012.

[39] C. S. Wallace, J. C. Champion, and G. A. Truskey, "Adhesion and function of human endothelial cells co-cultured on smooth muscle cells," Annals of Biomedical Engineering, vol. 35, no. 3, pp. 375-386, 2007.

[40] T. Korff, S. Kimmina, G. Martiny-Baron, and H. G. Augustin, "Blood vessel maturation in a 3-dimensional spheroidal coculture model: direct contact with smooth muscle cells regulates endothelial cell quiescence and abrogates VEGF responsiveness," The FASEB Journal, vol. 15, no. 2, pp. 447-457, 2001.

[41] L.-M. Zhao, L.-J. Ma, L.-X. Zhang, and J.-Z. Wu, "Shenmai injection inhibiting the extracellular signal regulated kinaseinduced human airway smooth muscle proliferation in asthma," Chinese Journal of Integrative Medicine, vol. 16, no. 4, pp. 331336, 2010.

[42] Y. Mebratu and Y. Tesfaigzi, "How ERK1/2 activation controls cell proliferation and cell death is subcellular localization the answer?" Cell Cycle, vol. 8, no. 8, pp. 1168-1175, 2009. 


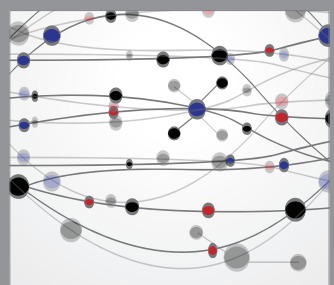

The Scientific World Journal
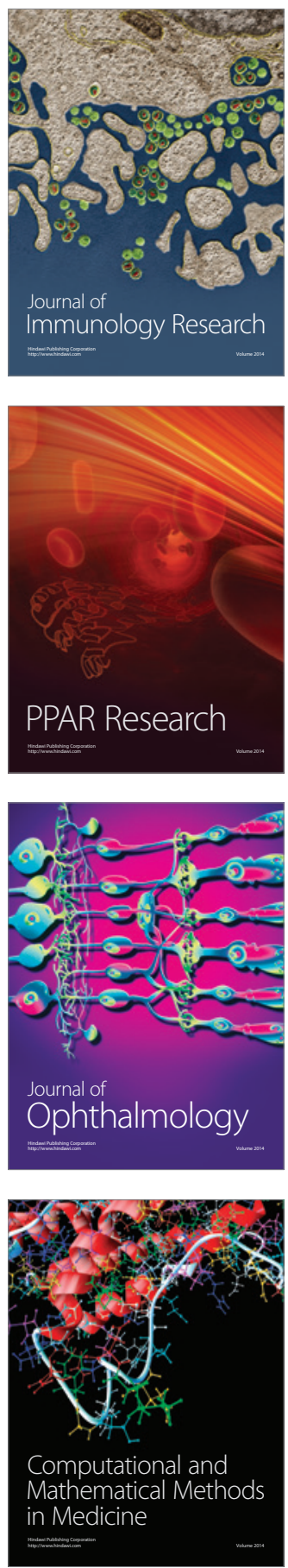

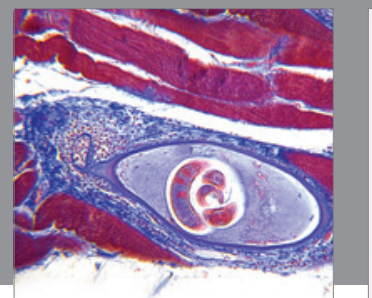

Gastroenterology

Research and Practice
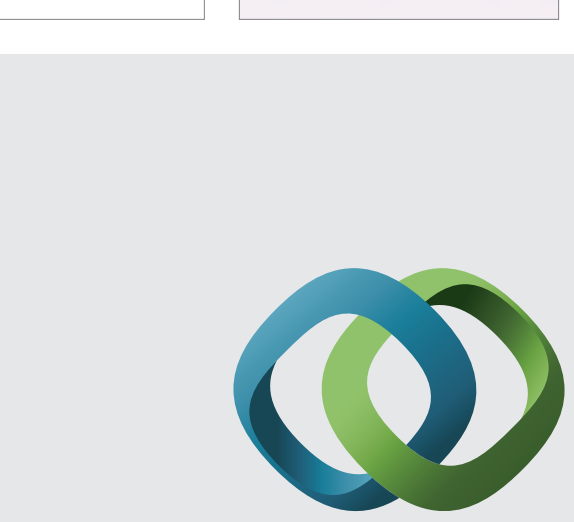

\section{Hindawi}

Submit your manuscripts at

http://www.hindawi.com
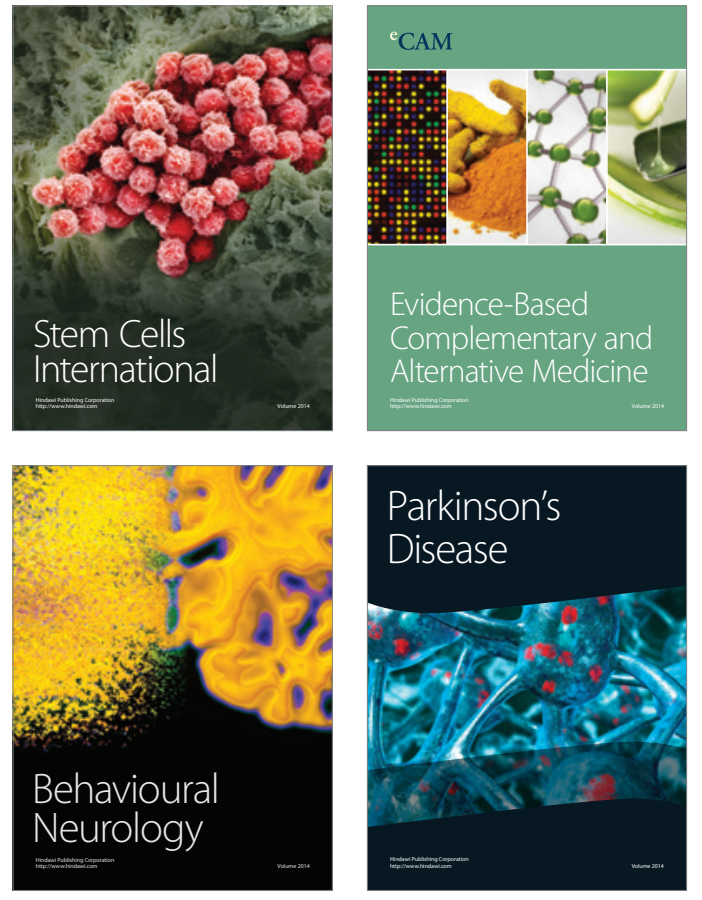
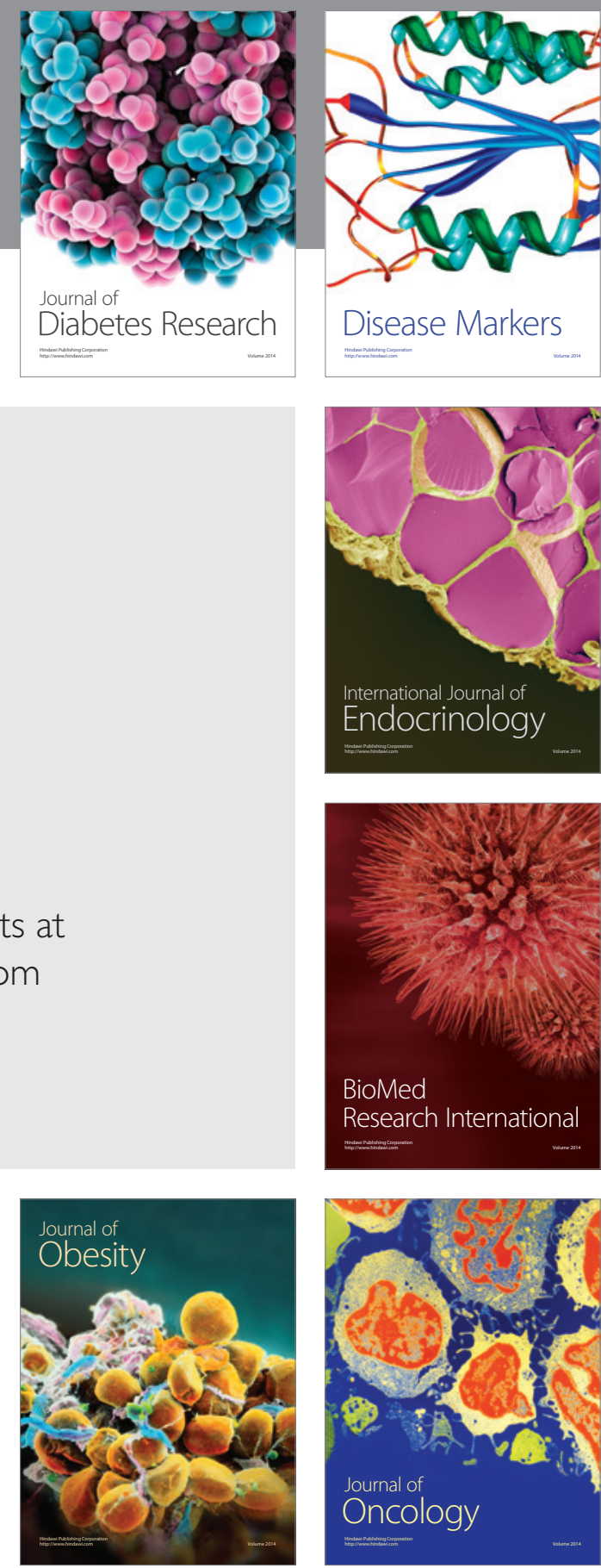

Disease Markers
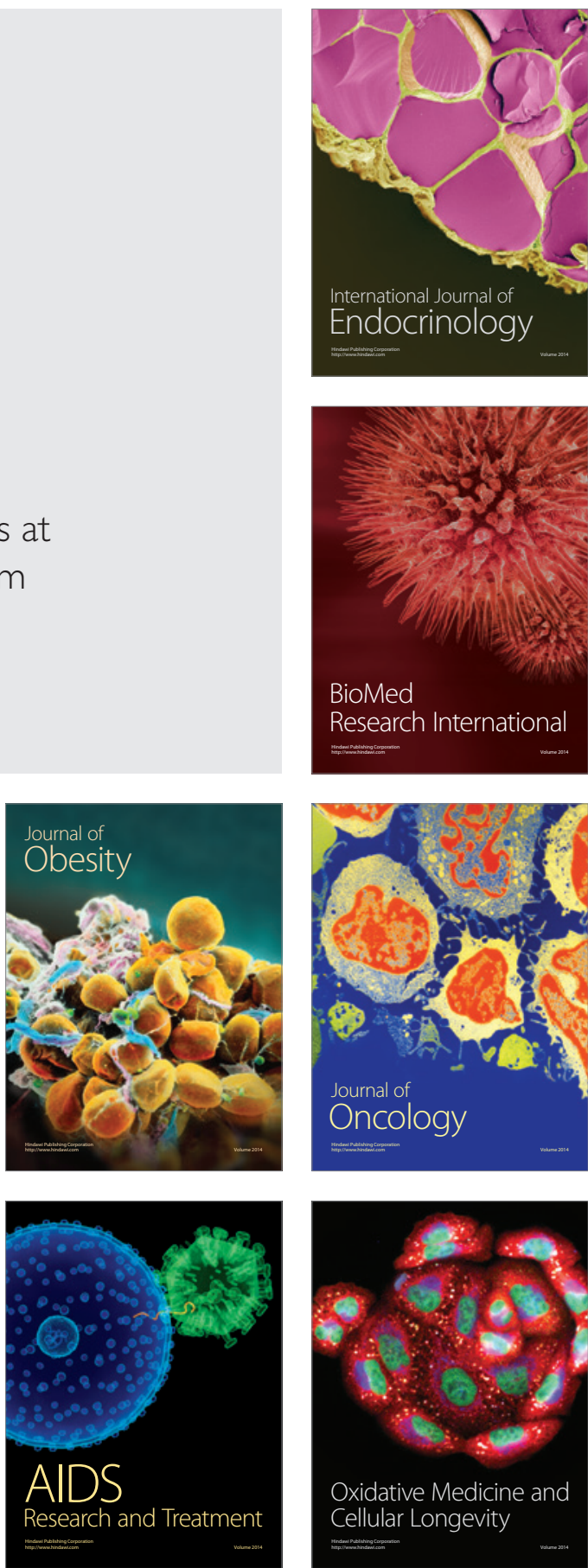